\title{
Erratum
}

Arch Pharm Res Vol 27, No 12, 1258-1262 (2004).

\section{Rolipram, a Phosphodiesterase 4 Inhibitor, Stimulates Osteoclast Formation by Inducing TRANCE Expression in Mouse Calvarial Cells}

Eun Sook Cho, Ja Heon Yu, Mi Sun Kim, and Mijung Yim

College of Pharmacy, Sookmyung Women's University, Seoul 140-742, Korea

In the above reference article, Fig. 1B, 1C, Fig. 2B, and Fig. 3A were printed unclearly. The better figures appear below.

Fig. 1B

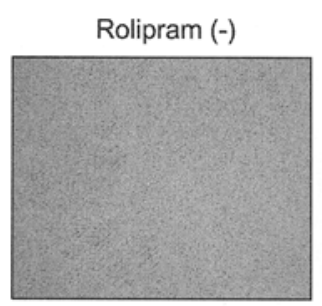

Rolipram (+)

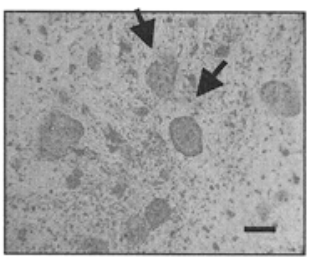

Fig. 1C

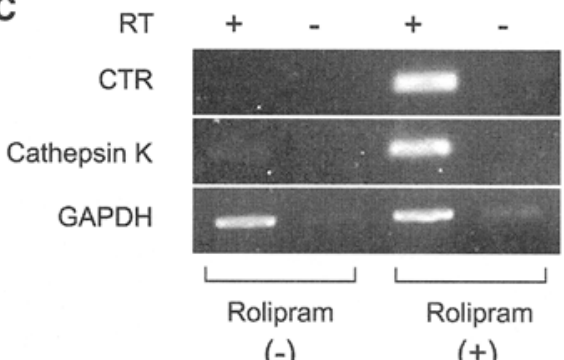

$(-)$

(+)
Fig. 2B

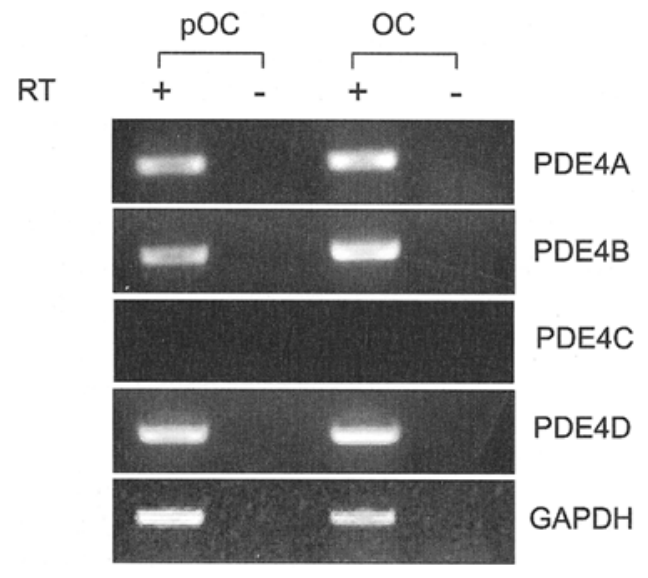

Fig. 3A

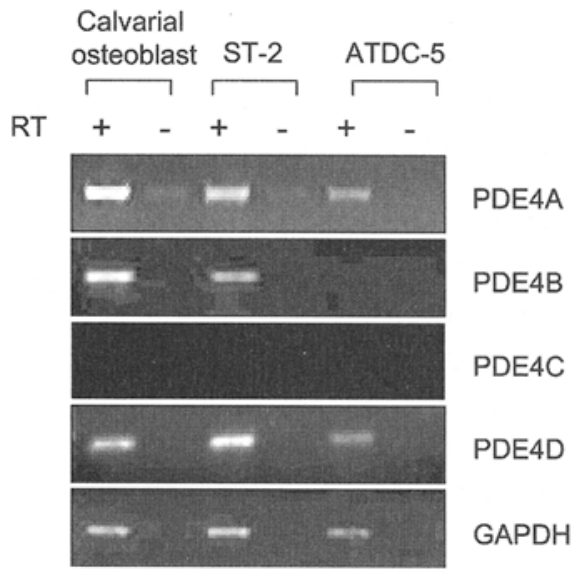

